\title{
Bioactive Cements: From Biological Properties to Clinical Applications ${ }^{\dagger}$
}

\author{
Gianni Di Giorgio 1, *, Alessandro Salucci ${ }^{1}$, Valeria Luzzi ${ }^{1}$, Carlo Rengo ${ }^{2}$, Michela Relucenti ${ }^{3}$, \\ Antonella Polimeni ${ }^{1}$, Maurizio Bossù ${ }^{1}$ \\ 1 Department of Oral and Maxillofacial Science, Sapienza University of Rome, 00185 Rome, Italy; \\ alessandro.salucci@uniroma1.it (A.S.); valeria.luzzi@uniroma1.it (V.L.); antonella.polimeni@uniroma1.it \\ (A.P.); maurizio.bossu@uniroma1.it (M.B.) \\ 2 Department of Prosthodontics and Dental Materials, School of Dental Medicine, University of Siena, 53100 \\ Siena, Italy; carlorengo@alice.it \\ 3 Department of Anatomy, Histology, Forensic Medicine and Orthopaedics, Sapienza University of Rome, \\ via Alfonso Borelli 50, 00161 Rome, Italy; michela.relucenti@uniroma1.it \\ * Correspondence: gianni.digiorgio@uniroma1.it \\ + Presented at 1st International Electronic Conference on Applied Sciences, 10-30 November 2020; Available \\ online: https://asec2020.sciforum.net/.
}

Published: 10 November 2020

\section{Oral Presentation:}

Bioactive materials basically contain two ceramic compounds, as tricalcium silicate and dicalcium silicate. These types of cements were successfully used in the endodontic field since their introduction in 1990 and possess several positive features as you can see here summarized. Particularly, the ability to interact with water and create an alkaline $\mathrm{pH}$ and calcium ions release, that, on turn, induce the formation of a superficial apatite layer and mineralization promotion, demonstrates their bioactivity. In addition, cell differentiation induction highlights their biocompatibility. The sealing, antibacterial and antifungal activities as well as the ability to either function as human tissues or encourage their regeneration strengthen their promising application in medicine and dentistry. Among bioactive materials, calcium silicate-based cements have achieved remarkable results in recent years, resulting in predictable, safe and successful therapies. Mineral Trioxide Aggregate (MTA) has been used in vital pulp therapy, in repair of root perforations or resorption, as root-end filling material in surgical endodontics and, more currently, in regenerative endodontic therapy. This hydraulic calcium silicate cement has a formulation similar to Portland cement with the addition of bismuth oxide, that is responsible for its radiopacity. MTA is composed by hydrophilic particles, that, once hydrated in a wet environment, develops a hard compound consisting mainly of tricalcium silicate, tricalcium aluminate, tricalcium oxide and silicate oxide. Despite its excellent qualities, MTA has some negative aspects related to its long setting time, difficult handling, discoloration of treated teeth and high cost. Therefore, to overcome these aforementioned drawbacks, a new bioceramic cement was recently introduced and applied mostly in the endodontics field, namely Biodentine. The latter is an innovative cement composed mainly of tricalcium silicate, calcium carbonate, used as a filler, and zirconium oxide, used as a radiopacifier. It's presented as powder and liquid that, after mixing, harden quickly thanks to the presence of calcium chloride in the liquid, that, on turn, reduces the setting time. From a clinical point of view, Biodentine showed similar applications to MTA, such as endodontic repairs and vital pulp therapy. Moreover, the same material demonstrated greater resistance to compression and flexion than other tricalcium silicate-based cements as well as sealing properties, bonding strength and a great release of calcium ions. Unlike MTA, Biodentine exhibited better handling, reduced setting time and color stability, since the absence of bismuth oxide which has been considered as the cause of dental discoloration. In order to support calcium silicate-based cements successful use 
in the clinical field, the aim of the present in vitro study was to demonstrate biocompatibility and antibiofilm properties of MTA and Biodentine. Both cements were mixed according to the manufacturer's instructions and were shaped in sterile rubber molds to obtain disks of $8 \mathrm{~mm}$ in diameter and $2 \mathrm{~mm}$ in thickness. After mixing, samples were kept in wet condition at $37^{\circ} \mathrm{C}$ for 24 hours to reach complete setting. Then, the specimens were used for the morphological, cytological and antimicrobical analyses. Human osteogenic sarcoma cells (Saos-2) were cultured and plated with cement extracts at ratio of 1:1,1:2, 1:4 or 1:8 dilutions. Cell viability was determined by using MTT assay and Reactive Oxygen Species (ROS) measurements and Immunofluorescence microscopy analysis were conducted on the cells exposed to cement extracts at 1:1 and 1:4 dilutions. The latter analysis aimed at evaluating the adhesion capability of cells to the cements. In addition, VpSEM and dEDS analysis were conducted on Human osteogenic sarcoma cells seeded on disks made up of both evaluated cements and Crystal Violet staining assay was used to assess the biofilm forming ability of Streptococcus mutans on the disks surfaces. MTT assay showed that cells had a dose-dependent cytotoxicity to both analyzed cements, at any time points. In particular, cells exposed to MTA extract showed a better cell vitality than those grown in the presence of the Biodentine one. Reactive Oxygen Species (ROS) measurements indicated that both materials were responsible for an increase of oxidative stress in Saos-2 cells in a dose-dependent manner, although, this appeared higher for the case of cells exposed to Biodentine extracts with respect to those exposed to MTA. These results suggest that the production of ROS could be the basis of different observed vitality. The Immunofluorescence analysis showed that the cells grown in the presence of the MTA extract, at all evaluated dilutions, demonstrated their classic elongated shape, with actin mainly organized in stress fibers, and an aspect highly similar to the control cells (treated only with the culture medium) as you can see in Figure A. Whereas, in the presence of Biodentine extract, the cells presented an altered morphology, more evident in case of undiluted condition. Moreover, in presence of extract at high dilution rate, the morphology was very similar to the control sample. To analyze the adhesion capability to the studied dental materials, cells were fixed and then marked with a specific antibody against one of the main components of the adhesion plaques, the focal adhesion kinase. The morphological analysis revealed that in presence of undiluted extract of Biodentine the adhesion plaques were almost absent, as observed in Figure B. Whereas, when the eluate was less concentrated, they become more evident, although lesser in number and in size than in presence of MTA. These results seemed to suggest a dose-dependent effect for Biodentine and a different behavior of the cells in terms of adhesion to the two types of evaluated cement. To evaluate the biocompatibility of Saos-2 cells grown directly on the ProRoot MTA or Biodentine disks, an additional set of MTT assay was performed at different times. The results obtained showed that the cells were able to adhere and grow at all evaluated time points. However, cells seeded on MTA presented a higher degree of biocompatibility compared to Biodentine, in agreement with the results obtained by MTT assay in the presence of both cement extracts. Moreover, the morphological analysis revealed that on the surface of MTA disks, Saos-2 cells were observed as distinct, wellpreserved, spheroid-shaped entities. On the other hand, the surface of the Biodentine disks showed spheroidal aggregates formed by 6-7 cellular elements. At higher magnification, Saos-2 cells grown on the surface of MTA disks, expressed blebs and microvilli on the surface of the apical domain; in addition, cells appeared well attached onto disks surface with their lamellipods, pseudopods and philopods. Considering the overall morphology, it seemed that these cells were perfectly viable. On the contrary, on the surface of the Biodentine disks rare adherent cells could be detected, with few scattered and short microvilli on the surface of the apical domain, and numerous pluricellular aggregates. Finally, Antiobiofilm activity analysis demonstrated that in the earliest phases, the adhesion of bacterial cells to Biodentine disks was not complete, while the surface of MTA samples resulted more covered after only 4 hours of exposition, as it can be observed in figure A. After 24 hours of growing, the few $\mathrm{S}$. mutans cells adhered on Biodentine disks, were able to build a mature biofilm, while on MTA samples their growth was inhibited, revealing a remarkable antibacterial effect with a decrease in biofilm biomass, as shown in figure B. According to the results of this in vitro study, both evaluated cements demonstrated to be biocompatible, although MTA has a cellular 
viability greater than that of Biodentine, and Saos-2 cells have a different behavior in terms of adhesion towards the two types of evaluated cements, suggesting a dose-dependent effect for Biodentine. Moreover, inhibition of superficial colonization as well as biofilm forming ability of S. mutants were successfully obtained with both evaluated cements, even though MTA demonstrated a more efficient antibiofilm effect than Biodentine, after 24 hours of observation. Therefore, although both cements proved to be biocompatible and to possess antibiofilm properties., MTA would seem to perform slightly better and could be considered as the gold standard material in the endodontic procedures. To corroborate the obtained in vitro results by the present study, I'd like to show two clinical cases developed at the Pediatric Dentistry Unit of Sapienza University of Rome. According to our clinical experience, bioceramic cements were successfully used in the endodontic treatment of deciduous teeth as well as permanent elements with immature root. As you can see in case 1, pulpotomy of a deciduous tooth was performed using MTA providing good clinical results over time. On the other hand, in case 2, a pulpotomy of a permanent tooth with immature roots was conducted with MTA and demonstrated stable results after 2 years of follow-up, allowing the formation of the dental root as well as the increase of root walls thickness.

Keywords: Antibiofilm properties; Bioactive cements; Biocompatibility; Biodentine; Endodontic procedures; MTA.

Publisher's Note: MDPI stays neutral with regard to jurisdictional claims in published maps and institutional affiliations.

(C) 2020 by the authors. Submitted for possible open access publication under the terms and conditions of the Creative Commons Attribution (CC BY) license

(http://creativecommons.org/licenses/by/4.0/). 